\title{
Age-related Changes of Macular Ganglion Cell-inner Plexiform Layer Thickness in Korean Elderly Subjects
}

\author{
Yun Ji Lee, Yung Ju Yoo, Sang Beom Han \\ Department of Ophthalmology, Kangwon National University School of Medicine, Chuncheon, Korea
}

\begin{abstract}
Purpose: We sought to establish normative ranges of the ganglion cell-inner plexiform layer (GCIPL) thickness using spectral-domain optical coherence tomography in Korean elderly individuals and to identify factors that influence GCIPL thickness. Methods: We conducted a retrospective, observational study of 114 healthy subjects (75 years old or older) who underwent comprehensive ophthalmic examinations at a single institution. GCIPL thickness was measured with the Cirrus spectral-domain optical coherence tomography system and automatic segmentation. Subjects were divided into two age groups: those younger than 80 years and those 80 years or older, respectively. A cross-sectional analysis was adopted to evaluate associations of GCIPL thickness with sex, age, intraocular pressure, optic disc rim area, axial length, spherical equivalent (SE) refractive errors, astigmatism, and body mass index.

Results: The average and minimum GCIPL thicknesses were $80.3 \pm 5.6 \mu \mathrm{m}$ and $76.3 \pm 5.9 \mu \mathrm{m}$, respectively. The GCIPL thickness was significantly lower in the older group than in the younger group in the inferior, inferonasal, and inferotemporal segments (all $p<0.01$ ). A thinner average GCIPL thickness was strongly associated with increasing age $(\beta=-2.87, p=0.021)$ and thinner circumpapillary retinal nerve fiber layer thickness $(\beta=2.87, p<0.001)$ in all segments.

Conclusions: GCIPL thickness decreased with age globally and in all segments, even after 75 years of age. Thinner GCIPL was associated with older age and thinner circumpapillary retinal nerve fiber layer. Age-related changes should be considered when using GCIPL thickness to assess glaucoma and other optic neuropathies characterized by retinal ganglion cell loss.
\end{abstract}

Key Words: Optical coherence tomography, Retina, Retinal ganglion cells

Optical coherence tomography (OCT) is the most widely used noninvasive imaging technique currently available for ocular structure evaluation [1,2]. With the advent of spectral-domain OCT (SD-OCT) technology with in-

Received: February 7, 2020 Final revision: May 8, 2020

Accepted: May 28, 2020

Corresponding Author: Yung Ju Yoo, MD. Department of Ophthalmology, Kangwon National University Hospital, Kangwon National University School of Medicine, 156 Baengnyeong-ro, Chuncheon 24289, Korea. Tel: 82-33-258-2196, Fax: 82-33-258-9138, E-mail: yyjuuuu@kangwon.ac.kr creased axial resolutions and faster scanning speeds relative to earlier-generation time-domain OCT systems, clinical practice has improved [3].

Along with the disc and retinal nerve fiber layer (RNFL) analysis, the ganglion cell-inner plexiform layer (GCIPL) thickness, which reflects the density of the ganglion cell body and the amount of dendrite loss, has been a focus of interest in recent studies. With advancements in technology, the adoption of a macular ganglion cell analysis (GCA) algorithm by SD-OCT had further enabled detailed GCIPL measurements to be performed in the macular region $[4,5]$. 
GCIPL measurements have been actively studied and clinically used in glaucoma. Macular GCA has supported improved evaluations of glaucoma when compared with RNFL analysis and has demonstrated good reproducibility in GCIPL measurements [6-8]. Macular inner retinal layer/ GCIPL thickness has been recognized as a sensitive indicator for the assessment of optic nerve damage, especially in glaucoma [8], optic neuritis, and ischemic optic neuropathy [8-11]. The assessment of GCIPL damage may be a useful adjunct for distinguishing compressive optic neuropathy from glaucomatous optic neuropathy when the results of optic disc and visual field examinations are inconclusive [12]. In terms of detecting retinal thickness changes in patients with demyelinating diseases such as multiple sclerosis, the use of average and minimum GCIPL measurements showed significantly better sensitivity than the adoption of temporal circumpapillary RNFL (cpRNFL) thickness in a previous study [13]. Larrea et al. [6] revealed that GCIPL thickness is capable of detecting early axonal damage in patients with nonarteritic anterior ischemic optic neuropathy in the acute phase, which could not be detected by cpRNFL.

The aforementioned diseases are common in elderly individuals. However, GCIPL thickness and its determinants within the general elderly population have not been widely studied [2,14-17]. This study therefore sought to assess GCIPL thickness values by applying the GCA algorithm on SD-OCT images to provide normative data for GCIPL thickness in an Korean elderly population. This study also evaluated the influences of age, sex, and ocular parameters on GCIPL thickness.

\section{Materials and Methods}

\section{Ethics statement}

The institutional review board of Kangwon National University Hospital approved this study (KNUH-2019-01005-001). This study was carried out in accordance with the recommendations of the Declaration of Helsinki for biomedical research involving human subjects. Informed consent was not gathered as patient records and information were anonymized and de-identified prior to undergoing analysis.

\section{Patients}

Patients older than 70 years of age who visited the ophthalmology clinic of Kangwon National Hospital between January 2016 and June 2018 were retrospectively reviewed. SD-OCT is a standard examination modality at Kangwon National Hospital for all adults presenting with visual disturbance. Eligible patients included those with best-corrected visual acuities of at least 20 / 25 and who were confirmed to have no ocular diseases other than low to medium refractive errors and dry eye syndrome. Only patients with no previous history of ocular surgery other than cataract surgery were included in this investigation. All subjects had an intraocular pressure (IOP) of $21 \mathrm{mmHg}$ or less and a normal-appearing optic disc, which was defined as an optic disk with the absence of glaucomatous optic neuropathy, pallor, or swelling of the optic disc. When glaucomatous optic neuropathy was suspected, the diagnosis was confirmed by visual field testing using standard automated perimetry (24-2 Swedish interactive thresholding algorithm) and the Humphrey Field Analyzer II 750 (Carl Zeiss Meditec, Dublin, CA, USA). For all patients, fundus photographs were evaluated by two neuro-ophthalmology specialists and no glaucomatous or neuro-ophthalmic optic nerve abnormalities were confirmed. The definition of a normal optic nerve head appearance was as follows: 1) the vertical cup-disc ratio was between 0.1 and $0.4 ; 2$ ) asymmetry of the cup-disc ratio was less than 0.2 ; and 3) no evidence of focal enlargement of the cup, superficial splinter hemorrhage, translucency of the neuroretinal rim, or development of vessel overpass was observed. When the opinions of the two neuro-ophthalmology specialists differed, another glaucoma specialist reviewed the pertinent photo; if all three specialists' conclusions remained out of alignment, the eye was excluded.

Each subject underwent a complete ophthalmic examination, which included visual acuity and refraction assessments, slit-lamp biomicroscopy, noncontact tonometry (ICT-800; KOWA, Tokyo, Japan), and fundus photography (EOS D60 digital camera; Canon, Utsunomiya, Japan). If the IOP as measured by the noncontact tonometry or rebound tonometry was greater than $21 \mathrm{mmHg}$, an additional test was performed with Goldmann applanation tonometry (Haag-Streit AG, Bern, Switzerland) to confirm.

For this study, we excluded patients with a high refractive error, defined as a spherical equivalent (SE) refractive 
error exceeding \pm 6.00 diopters (D) or astigmatism exceeding $3.00 \mathrm{D}$, or evidence of vitreoretinal diseases or optic nerve abnormality. Patients with a history of amblyopia, who were suspected of having glaucoma; or who had general conditions capable of causing changes to the macular thickness (e.g., diabetes mellitus, leukemia, acquired immunodeficiency syndrome, dementia, or multiple sclerosis) were also excluded [18].

\section{SD-OCT examination}

The GCIPL and cpRNFL data were collected using the Cirrus OCT system (Carl Zeiss Meditec), the details of which have been described previously $[4,8,18-20]$. After pharmacologic dilation of the pupil, one $200 \times 200$ optic disc cube scan and one $200 \times 200$ macula cube scan were acquired in both eyes of the same subject [17]. Only high-quality scans were included in the analysis, which included those with a signal strength of greater than 6 and without motion artifacts, involuntary eye movement, overt misalignment or decentration, or algorithm segmentation failure, respectively [20]. Intraretinal findings and abnormalities at the vitreoretinal interface such as epiretinal membranes were identified using the high-definition fiveline raster scan capability of the Cirrus OCT system. According to the cpRNFL and optic nerve head parameters, the Cirrus OCT software automatically tracked the central point of the optic nerve head and then, to calculate the RNFL thickness at each point of the circle, it extracted a 3.46-mm-diameter circumpapillary circle [4]. Using these data, the Cirrus OCT system provided further data on the 12-o'clock-hour thickness, four-quadrant thickness, and global $360^{\circ}$ average thickness of the cpRNFL [21].

The GCA algorithm of the Cirrus SD-OCT system included in the 6.0 software version has been described in detail elsewhere $[4,19]$. Briefly, the GCIPL thickness was measured in the area defined by two elliptical annuli centered on the macula. The inner ring has a vertical diameter of $1 \mathrm{~mm}$ and a horizontal diameter of $1.2 \mathrm{~mm}$. The outer ring has a vertical diameter of $4 \mathrm{~mm}$ and a horizontal diameter of $4.8 \mathrm{~mm}$ [17]. The inner-ring excluded the foveal area wherein the GCIPL was too thin to detect [4]. Meanwhile, the outer-ring size was selected to include the area wherein the GCIPL was thickest in a normal population. The GCA algorithm detects the outer boundaries of both the RNFL and inner plexiform layer and the distance be- tween these two segmentations is defined as the GCIPL $[4,21]$.

\section{Statistical analysis}

Statistical analysis was performed using the IBM SPSS Statistics ver. 21.0 (IBM Corp., Armonk, NY, USA). Although data were acquired from both eyes, only those from one randomly selected eye per participant were adopted for statistical analysis. Comparisons of macular thickness between the two age groups were conducted using the unpaired t-test with Bonferroni adjustment. Results were considered statistically significant when original the $p$-value was less than 0.0083 ( 0.05 / 6 Bonferroni adjustment). Associations between the macular GCIPL thickness and the following seven factors were assessed: sex, age, laterality, IOP, astigmatism, SE refractive errors, and the average cpRNFL. Univariate linear regression analyses were conducted, followed by multivariate linear regression models for factors with an association at a $p$-value of 0.05 or less in the univariate analysis. To determine how the factors accounted for variability, we converted the coefficient of determination into a percentage. The cutoff for statistical significance was set at a $p$-value of less than 0.05 . Except where stated otherwise, data are presented as mean \pm standard deviation values. One eye from each study patient was randomly chosen for inclusion in the statistical analysis.

\section{Results}

\section{Baseline characteristics}

The demographics and ocular characteristics of the 114 study participants are described in Table 1. The average age of the study population was $80.9 \pm 3.7$ years, ranging from 75 to 90 years, and 63 participants $(55.2 \%)$ were male. The mean IOP was $11.3 \pm 2.1 \mathrm{mmHg}$ and SE refractive errors were $+0.73 \pm 1.68 \mathrm{D}$. The mean rim area of the optic nerve was $2.07 \pm 0.46 \mathrm{~mm}^{2}$. Patient ages ranged from 75 to 90 years and were divided into two age groups: the younger group consisted of subjects ranging from 75 to 79 years of age (n $=52,20$ males and 32 females), while the older group consisted of subjects ranging from 80 to 90 years of age ( $\mathrm{n}=$ 62, 31 males and 31 females). In a subgroup analysis based 
Table 1. Clinical characteristics of the 114 subjects

\begin{tabular}{lc}
\hline Characteristics & Value \\
\hline Mean age (yr) & $80.9 \pm 3.7(75-90)$ \\
Sex (male : female) & $51: 63$ \\
Laterality (right : left) & $60: 54$ \\
Intraocular pressure (mmHg) & $11.3 \pm 2.1(8-19)$ \\
Visual acuity (logMAR) & $0.03 \pm 0.08(-0.18$ to 0.22$)$ \\
SE refractive errors (D) & $0.73 \pm 1.68(-3.63$ to 5.13$)$ \\
Axial length (mm) & $23.48 \pm 0.83(21.2-25.2)$ \\
Astigmatism (D) & $1.40 \pm 0.81(0.00-3.00)$ \\
BMI ${ }^{*}\left(\mathrm{~kg} / \mathrm{m}^{2}\right)$ & $24.08 \pm 3.20(15.82-38.87)$ \\
$\quad<21$ & $14(12.3)$ \\
$21-26.9$ & $80(70.2)$ \\
$27-29.9$ & $10(8.8)$ \\
$\geq 30$ & $5(4.4)$ \\
Rim area $\left(\mathrm{mm}^{2}\right)$ & $2.07 \pm 0.46(1.34-4.15)$ \\
\hline
\end{tabular}

Values are mean \pm standard deviation (range), number, or number (\%). $\log$ MAR $=$ logarithm of the minimum angle of resolution; SE = spherical equivalent; $\mathrm{D}=$ diopter; $\mathrm{BMI}=$ body mass index. *Missing data: five for BMI. on age, best-corrected visual acuity, IOP, SE refractive errors, and axial length were not significantly different between the younger and older age groups (all $p>0.2$ ).

\section{cpRNFL and total macular thickness measurements}

Table 2 shows the mean cpRNFL and total macular thickness globally and in different segments of all subjects and subgroups divided by age and sex. The average global cpRNFL thickness was $91.2 \pm 8.4 \mu \mathrm{m}$, ranging from 66.9 to $116 \mu \mathrm{m}$ (Table 2). The average and inferior segment cpRNFL thicknesses showed significant differences between the two age groups ( $p=0.01$ and 0.005 after Bonferroni correction, respectively) and were thinner in the older age group. The average macular thickness of all the subjects was $313.0 \pm 12.3 \mu \mathrm{m}$, ranging from 277.6 to 371.1 $\mu \mathrm{m}$. The inferior outer segment total macular thickness displayed a significant difference between the two age groups ( $p=0.005$ after Bonferroni correction) (Table 2).

Table 2. Circumpapillary retinal nerve fiber layer and macular retinal thickness according to age and sex

\begin{tabular}{|c|c|c|c|c|}
\hline & $\begin{array}{c}\text { Total } \\
(\mathrm{n}=114)\end{array}$ & $\begin{array}{l}<80 \text { years } \\
(\mathrm{n}=52)\end{array}$ & $\begin{array}{l}\geq 80 \text { years } \\
(\mathrm{n}=62)\end{array}$ & $p$-value \\
\hline \multicolumn{5}{|c|}{$\begin{array}{l}\text { Retinal nerve fiber layer thickness } \\
\text { in quadrants }(\mu \mathrm{m})\end{array}$} \\
\hline Average & $91.2 \pm 8.4$ & $93.8 \pm 8.1$ & $89.0 \pm 8.1$ & $0.002^{*}$ \\
\hline Superior & $111.9 \pm 13.5$ & $113.8 \pm 12.3$ & $110.2 \pm 14.4$ & 0.164 \\
\hline Temporal & $70.7 \pm 12.5$ & $72.1 \pm 11.5$ & $69.1 \pm 13.4$ & 0.207 \\
\hline Inferior & $116.2 \pm 15.4$ & $121.4 \pm 13.7$ & $111.8 \pm 15.5$ & $0.001^{*}$ \\
\hline Nasal & $66.9 \pm 9.4$ & $68.0 \pm 7.2$ & $65.9 \pm 10.7$ & 0.243 \\
\hline \multicolumn{5}{|c|}{ Macular retinal thickness $(\mu \mathrm{m})$} \\
\hline Center & $252.5 \pm 15.4$ & $252.3 \pm 15.4$ & $253.6 \pm 14.4$ & 0.646 \\
\hline Superior inner & $320.0 \pm 13.5$ & $321.4 \pm 16.6$ & $318.5 \pm 10.8$ & 0.27 \\
\hline Temporal inner & $312.1 \pm 14.6$ & $315.5 \pm 17.8$ & $309.4 \pm 10.7$ & 0.026 \\
\hline Inferior inner & $316.7 \pm 15.3$ & $320.0 \pm 15.2$ & $313.9 \pm 15.2$ & 0.035 \\
\hline Nasal inner & $323.0 \pm 14.3$ & $325.7 \pm 16.3$ & $320.9 \pm 12.2$ & 0.076 \\
\hline Superior outer & $280.2 \pm 16.0$ & $283.5 \pm 16.8$ & $277.4 \pm 14.8$ & 0.04 \\
\hline Temporal outer & $265.1 \pm 14.3$ & $268.4 \pm 14.1$ & $262.5 \pm 14.0$ & 0.03 \\
\hline Inferior outer & $265.8 \pm 13.2$ & $270.4 \pm 12.8$ & $262.1 \pm 12.5$ & $0.001^{*}$ \\
\hline Nasal outer & $295.3 \pm 12.8$ & $298.1 \pm 13.9$ & $292.6 \pm 11.3$ & 0.022 \\
\hline
\end{tabular}

Values are presented as mean \pm standard deviation.

${ }^{*}$ Factors with statistical significance. 
Table 3. Macular GCIPL thickness obtained from the 114 subjects in each of the six subfields

\begin{tabular}{|c|c|c|c|c|c|c|c|c|c|c|}
\hline \multirow[b]{2}{*}{ GCIPL $(\mu \mathrm{m})$} & \multicolumn{4}{|c|}{ Total $(n=114)$} & \multicolumn{3}{|c|}{ Male } & \multicolumn{3}{|c|}{ Female } \\
\hline & Total & $\begin{array}{c}<80 \mathrm{yr} \\
(\mathrm{n}=52)\end{array}$ & $\begin{array}{l}\geq 80 \mathrm{yr} \\
(\mathrm{n}=62)\end{array}$ & $p$-value & $\begin{array}{c}<80 \mathrm{yr} \\
(\mathrm{n}=20)\end{array}$ & $\begin{array}{l}\geq 80 \mathrm{yr} \\
(\mathrm{n}=31)\end{array}$ & $p$-value & $\begin{array}{c}<80 \mathrm{yr} \\
(\mathrm{n}=32)\end{array}$ & $\begin{array}{l}\geq 80 \mathrm{yr} \\
(\mathrm{n}=31)\end{array}$ & $p$-value \\
\hline Average & $80.3 \pm 5.6$ & $81.5 \pm 5.7$ & $79.1 \pm 5.5$ & $0.001^{*}$ & $81.6 \pm 4.8$ & $78.1 \pm 4.5$ & 0.012 & $82.0 \pm 5.6$ & $80.1 \pm 6.3$ & 0.036 \\
\hline Minimum & $76.3 \pm 5.9$ & $77.2 \pm 6.3$ & $75.2 \pm 6.0$ & 0.032 & $77.5 \pm 5.8$ & $75.1 \pm 4.3$ & 0.098 & $77.7 \pm 5.7$ & $75.3 \pm 7.4$ & 0.161 \\
\hline Superior & $80.9 \pm 6.2$ & $81.7 \pm 6.2$ & $80.1 \pm 6.4$ & 0.017 & $80.6 \pm 5.1$ & $78.4 \pm 5.5$ & 0.155 & $82.8 \pm 6.1$ & $81.8 \pm 6.8$ & 0.115 \\
\hline Superior temporal & $80.8 \pm 6.0$ & $81.7 \pm 6.5$ & $79.8 \pm 5.5$ & 0.013 & $81.8 \pm 6.5$ & $78.9 \pm 4.8$ & 0.078 & $82.0 \pm 6.3$ & $80.7 \pm 6.2$ & 0.105 \\
\hline Inferior temporal & $81.1 \pm 6.2$ & $82.7 \pm 6.2$ & $79.6 \pm 6.1$ & $0.001^{*}$ & $83.7 \pm 5.5$ & $78.9 \pm 5.6$ & $0.004^{*}$ & $82.3 \pm 6.2$ & $80.3 \pm 6.7$ & 0.039 \\
\hline Inferior & $77.4 \pm 6.6$ & $79.5 \pm 6.2$ & $75.4 \pm 6.5$ & $<0.001^{*}$ & $79.7 \pm 5.6$ & $74.5 \pm 5.3$ & $0.002^{*}$ & $79.7 \pm 6.2$ & $76.4 \pm 7.4$ & $0.004^{*}$ \\
\hline Inferior nasal & $79.7 \pm 6.4$ & $81.0 \pm 6.9$ & $78.3 \pm 6.3$ & $0.002^{*}$ & $80.8 \pm 5.9$ & $77.6 \pm 5.5$ & 0.055 & $81.7 \pm 6.6$ & $79.0 \pm 7.0$ & 0.020 \\
\hline Superior nasal & $82.4 \pm 5.9$ & $83.1 \pm 6.3$ & $81.4 \pm 6.1$ & 0.02 & $82.9 \pm 4.9$ & $80.5 \pm 5.6$ & 0.117 & $84.0 \pm 6.0$ & $82.4 \pm 6.4$ & 0.125 \\
\hline
\end{tabular}

Values are presented as mean \pm standard deviation.

GCIPL = ganglion cell-inner plexiform layer.

${ }^{*}$ Factors with statistical significance.

Table 4. Variation in the average macular ganglion cell-inner plexiform layer thickness according to demographic variables and ocular parameters

\begin{tabular}{|c|c|c|c|c|c|c|}
\hline \multirow{2}{*}{ Characteristics } & \multicolumn{3}{|c|}{ Univariate analysis } & \multicolumn{3}{|c|}{ Multivariate analysis } \\
\hline & $\beta$ & $95 \% \mathrm{CI}$ & $p$-value & $\beta$ & $95 \% \mathrm{CI}$ & $p$-value \\
\hline Male, sex & 0.929 & -1.099 to 2.958 & 0.366 & & & \\
\hline Age per 10 years older & $-4.302^{*}$ & $-6.903^{*}$ to $-1.702^{*}$ & $0.001^{*}$ & $-2.874^{*}$ & $-5.302^{*}$ to $-0.446^{*}$ & $0.021^{*}$ \\
\hline IOP per $1 \mathrm{mmHg}$ higher & 0.315 & -0.173 to 0.803 & 0.203 & & & \\
\hline $\begin{array}{l}\text { Average cpRNFL thickness per } 10 \mu \mathrm{m} \\
\quad \text { larger }\end{array}$ & $3.239^{*}$ & $2.166^{*}$ to $4.311^{*}$ & $<0.001^{*}$ & $2.869^{*}$ & $1.684^{*}$ to $4.053^{*}$ & $<0.001^{*}$ \\
\hline Rim area per $1 \mathrm{~mm}^{2}$ larger & $4.341^{*}$ & $0.01^{*}$ to $8.628^{*}$ & $0.047^{*}$ & 0.539 & -3.4636 to 4.515 & 0.789 \\
\hline Axial length per mm longer & -0.211 & -1.447 to 1.025 & 0.736 & & & \\
\hline SE refractive errors per $1 \mathrm{D}$ higher & -0.670 & -1.365 to 0.025 & 0.059 & & & \\
\hline Astigmatism per $1 \mathrm{D}$ higher & 0.579 & -0.889 to 2.046 & 0.435 & & & \\
\hline BMI per $1 \mathrm{~kg} / \mathrm{m}^{2}$ higher & -0.238 & -0.550 to 0.074 & 0.134 & & & \\
\hline
\end{tabular}

$\mathrm{CI}=$ confidence interval; $\mathrm{IOP}=$ intraocular pressure; $\mathrm{cpRNFL}=$ circumpapillary retinal nerve fiber layer; $\mathrm{SE}=$ spherical equivalent; $\mathrm{D}=$ diopters; $\mathrm{BMI}=$ body mass index.

${ }^{*}$ Factors with statistical significance.

\section{GCIPL thickness measurements}

Table 3 shows the average GCIPL thickness among different segments according to age and sex. The average GCIPL thickness was $80.3 \pm 5.6 \mu \mathrm{m}$ (range, 76.3 to $82.4 \mu \mathrm{m}$ ), with a median value of $80 \mu \mathrm{m}$, while the minimum GCIPL thickness was $76.3 \pm 5.9 \mu \mathrm{m}$ (range, 63 to $90 \mu \mathrm{m}$ ). Further, the average GCIPL thickness was $79.4 \pm 5.4 \mu \mathrm{m}$ in males and 82.3 $\pm 6.4 \mu \mathrm{m}$ in females (Table 3). The highest value was found in the superonasal segment and the lowest value was found in the inferior segment in both male and female individuals. For men, GCIPL thicknesses in the inferotemporal and inferior segments were higher among participants aged younger than 80 years than among those aged 80 years or older $(p=$ 0.004 and 0.002 after Bonferroni correction, respectively), while, for women, the GCIPL thickness was significantly different between the two age groups in the inferior segment ( $p=0.004$ after Bonferroni correction) (Table 3). 


\section{Correlations between GCIPL thickness and predictors}

Table 4 shows the results of univariate and multivariate linear regression analyses between the average GCIPL thickness and sex, age, IOP, average cpRNFL thickness, optic disc rim area, axial length, SE refractive errors, and body mass index. There was a statistically significant decline in the mean GCIPL thickness with age, cpRNFL, and rim area $(\beta=-4.302, p=0.001 ; \beta=3.239, p<0.001$; and $\beta$ $=4.341, p=0.047$, respectively) observable in the univariate analysis (Table 4 and Fig. 1A, 1B). The multivariate analysis revealed that age and average cpRNFL thickness was significantly associated with the GCIPL thickness $(\beta=$ -2.874; $p=0.021$ and $\beta=2.869 ; p<0.001$, respectively) (Table 4). Fig. 1 shows the relationship between the average GCIPL thickness and age.

Regarding the minimal GCIPL thickness, only the average cpRNFL was significantly associated with GCIPL thickness. Older age correlated with a decrease in the average GCIPL thickness of $4.30 \mu \mathrm{m}$ per decade $(p=0.001)$ as well as in all different segments ( $\beta$ range, -5.72 to -3.62 ; all $p<0.05)$. The thickness in all sectors was significantly associated with cpRNFL ( $\beta$ range, 2.67 to 3.67; all $p<0.001$ ). The change in macular sectorial GCIPL thickness in relation to cpRNFL thickness is shown in Fig. 1. Regardless of which segments were considered, there was no difference in the GCIPL thickness according to the IOP, axial length, or SE refractive error. Rim area was associated with the GCIPL thickness in the inferior and inferotemporal seg- ments $(\beta=7.16 ; p=0.005$ and $\beta=5.04 ; p=0.039$, respectively).

\section{Discussion}

Early identification of diseases of the retina and optic nerve has become increasingly accurate and easier to perform with the advent of SD-OCT, which offers new structural parameters including cpRNFL thickness and individual retinal layer thickness in the macula. Owing to the development of powerful segmentation algorithms, several studies have determined that GCIPL thickness as measured by SD-OCT appears to be a more appropriate marker of early retinal layer injury in acute optic nerve diseases when compared with cpRNFL thickness $[6,12]$ and is also useful in the differentiation of chronic optic neuropathy $[8,22]$. Using the results of this study as reference values, SD-OCT could be adopted as a diagnostic tool in retinal and optic nerve diseases in elderly patients.

This study provided normative ranges of GCIPL thickness in Korean elderly subjects using the Cirrus SD-OCT system and accompanying GCA algorithm. There were significant correlations observed between age and cpRNFL thickness and average GCIPL thickness. We did not demonstrate a significant correlation between the GCIPL thickness and other ocular parameters such as IOP, axial length, optic disc rim area, and body mass index among subjects. The average GCIPL thickness as measured using
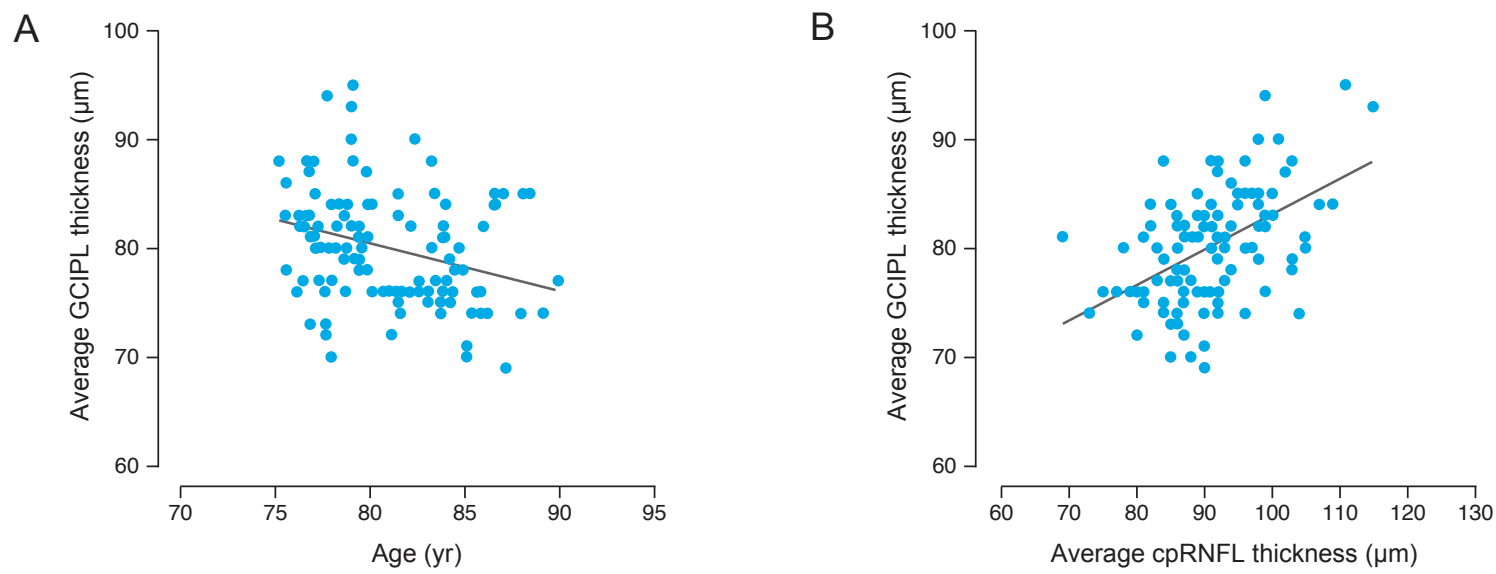

Fig. 1. Scatterplots and regression lines to estimate the effects of age and average circumpapillary retinal nerve fiber layer (cpRNFL) thickness on the average ganglion cell-inner plexiform layer (GCIPL) thickness. (A) A thinner average GCIPL thickness was strongly associated with increasing age $(\beta=-4.302, p=0.001)$ and $(\mathrm{B})$ a thinner $\mathrm{cpRNFL}$ thickness $(\beta=3.239, p<0.001)$. 
the Cirrus SD-OCT system in our study was $80.3 \pm 5.6$ $\mu \mathrm{m}$, which is slightly lower than values previously reported after using the same SD-OCT system $(80.8 \mu \mathrm{m}$ [16] and $82.8 \mu \mathrm{m}$ [14]). This discrepancy was due to the variation in the average age of subjects between these studies [14,16,17]. The mean age of subjects in our study was higher than those in previous investigations $[14,16,17]$. Interestingly, the larger the difference in the mean age was, the greater the difference noted in the GCIPL thickness was.

Our study revealed that age had a significant effect on the average GCIPL thickness in each segment. Specifically, a $0.29-\mu \mathrm{m}$ decrease in the average GCIPL thickness per year in elderly Korean subjects was associated with age. Previous studies suggested that the linear reduction in the GCIPL thickness is in the range of 0.14 to $0.30 \mu \mathrm{m} / \mathrm{yr}$ [14,16,23-25]. Prior histological studies also found that the ganglion cell layer (GCL) and associated axons (RNFL) are vulnerable to loss during aging, consistent with the results of our study [26]. The normative database for GCIPL thickness assessed using the Cirrus SD-OCT system consists of 284 healthy subjects of different ethnicities with a mean age of 46.5 years (range, 18 to 84 years) [18]. Another study reported an age-related linear loss of GCIPL in subjects ranging in age from 18 to 85 years. However, the numbers of older individuals in previous studies were relatively small $[17,18]$. Therefore, the results of the present study, which included many patients older than 70 years, have unique value.

Our study failed to reveal a significant sex difference in GCIPL thickness. Among adult populations, a few studies have identified sex differences in the inner sector macular thickness [14,19,27,28], whereas others have observed no relationship [16,29]. However, our study was limited in that it included only elderly patients with advanced age-related GCIPL thinning. Moreover, previous assessments have not analyzed the impact of sex on GCIPL thickness by age. The relationship between sex and GCIPL thickness measurements is hypothetical and should be verified through a longitudinal study.

There was a positive correlation observed between the GCIPL and cpRNFL thicknesses. Multiple regression analysis revealed that each micrometer decrease in the cpRNFL thickness corresponded with a $0.29-\mu \mathrm{m}$ decrease in the GCIPL thickness. As the cpRNFL is composed of axons of GCL, we could presume that a thinner GCL would result in a thinner cpRNFL and vice versa. Previous studies have also suggested that the GCIPL thickness is correlated with the cpRNFL thickness [14,17,30].

Interestingly, there was no correlation found between the GCIPL thickness and disc area. As the number of RGCs is determined by the number of axons [17,31,32], it can be seen that the number of axon projections within the RNFL is not correlated with the disc size [33-35]. There are controversies regarding the association between disc size, RNFL thickness, and the number of axons [17]. Although some studies have not observed any association between disc size and RNFL thickness, others identified a positive correlation between these two [36,37]. Discrepancies in the disc size-dependent effect among previous studies may be due to the large anatomical variations in the number of RGCs and in the optic disc size [17].

There are several limitations in this study that need to be considered. First, as it was a retrospective cross-sectional study, a degree of selection bias might exist. Caution is therefore needed before extrapolating these results to the general population. A larger prospective cohort study is necessary to confirm our findings. Second, we included patients only in their 70 s and 80 s. Further assessments should be carried out involving patients of a wider range of ages to reduce the bias that masks age-specific differences. However, our study included enough subjects in each age group relative to in previous studies. Since this study was hospital-based, it has a limitation of not completely covering the general population. However, with the strict inclusion and exclusion criteria, any bias in this regard was eliminated as much as possible; we included patients who had only low- to medium-level refractive errors and dry eye syndrome, which presents a high degree of prevalence even in the general population.

In conclusion, the present hospital-based study described normative ranges of the GCIPL thickness as measured using the Cirrus SD-OCT system in a relatively large number of Korean adults. The thinner GCIPL thickness in the eyes of Korean elderly individuals was independently associated with aging and reduced cpRNFL thickness. These factors should be considered when interpreting the GCIPL thickness based on Cirrus SD-OCT system measurements for the evaluation of glaucoma and other optic neuropathies. 


\section{Conflict of Interest}

No potential conflict of interest relevant to this article was reported.

\section{Acknowledgements}

This study was supported by 2020 Kangwon National University Hospital Grant.

\section{References}

1. Huang D, Swanson EA, Lin CP, et al. Optical coherence tomography. Science 1991;254:1178-81.

2. Zhang X, Francis BA, Dastiridou A, et al. Longitudinal and cross-sectional analyses of age effects on retinal nerve fiber layer and ganglion cell complex thickness by Fourier-domain OCT. Transl Vis Sci Technol 2016;5:1.

3. Drexler W, Sattmann H, Hermann B, et al. Enhanced visualization of macular pathology with the use of ultrahigh-resolution optical coherence tomography. Arch Ophthalmol 2003;121:695-706.

4. Kim KE, Park KH, Jeoung JW, et al. Severity-dependent association between ganglion cell inner plexiform layer thickness and macular mean sensitivity in open-angle glaucoma. Acta Ophthalmol 2014;92:e650-6.

5. Kim KE, Yoo BW, Jeoung JW, Park KH. Long-term reproducibility of macular ganglion cell analysis in clinically stable glaucoma patients. Invest Ophthalmol Vis Sci 2015;56:4857-64.

6. Larrea BA, Iztueta MG, Indart LM, Alday NM. Early axonal damage detection by ganglion cell complex analysis with optical coherence tomography in nonarteritic anterior ischaemic optic neuropathy. Graefes Arch Clin Exp Ophthalmol 2014;252:1839-46.

7. Weinreb RN, Aung T, Medeiros FA. The pathophysiology and treatment of glaucoma: a review. JAMA 2014;311:190111.

8. Mwanza JC, Durbin MK, Budenz DL, et al. Glaucoma diagnostic accuracy of ganglion cell-inner plexiform layer thickness: comparison with nerve fiber layer and optic nerve head. Ophthalmology 2012;119:1151-8.

9. Nouri-Mahdavi K, Nowroozizadeh S, Nassiri N, et al. Macular ganglion cell/inner plexiform layer measurements by spectral domain optical coherence tomography for detection of early glaucoma and comparison to retinal nerve fiber layer measurements. Am J Ophthalmol 2013;156:1297307.

10. Sato S, Hirooka K, Baba T, et al. Correlation between the ganglion cell-inner plexiform layer thickness measured with cirrus HD-OCT and macular visual field sensitivity measured with microperimetry. Invest Ophthalmol Vis Sci 2013;54:3046-51.

11. Kim HJ, Lee SY, Park KH, et al. Glaucoma diagnostic ability of layer-by-layer segmented ganglion cell complex by spectral-domain optical coherence tomography. Invest Ophthalmol Vis Sci 2016;57:4799-805.

12. Lee EJ, Yang HK, Kim TW, et al. Comparison of the pattern of retinal ganglion cell damage between patients with compressive and glaucomatous optic neuropathies. Invest Ophthalmol Vis Sci 2015;56:7012-20.

13. Gonzalez-Lopez JJ, Rebolleda G, Leal M, et al. Comparative diagnostic accuracy of ganglion cell-inner plexiform and retinal nerve fiber layer thickness measures by Cirrus and Spectralis optical coherence tomography in relapsing-remitting multiple sclerosis. Biomed Res Int 2014;2014:128517.

14. Koh VT, Tham YC, Cheung CY, et al. Determinants of ganglion cell-inner plexiform layer thickness measured by high-definition optical coherence tomography. Invest Ophthalmol Vis Sci 2012;53:5853-9.

15. Cheung CY, Ong YT, Hilal S, et al. Retinal ganglion cell analysis using high-definition optical coherence tomography in patients with mild cognitive impairment and Alzheimer's disease. J Alzheimers Dis 2015;45:45-56.

16. Huo YJ, Guo Y, Li L, et al. Age-related changes in and determinants of macular ganglion cell-inner plexiform layer thickness in normal Chinese adults. Clin Exp Ophthalmol 2018;46:400-6.

17. Mwanza JC, Durbin MK, Budenz DL, et al. Profile and predictors of normal ganglion cell-inner plexiform layer thickness measured with frequency-domain optical coherence tomography. Invest Ophthalmol Vis Sci 2011;52:78729.

18. Knight OJ, Girkin CA, Budenz DL, et al. Effect of race, age, and axial length on optic nerve head parameters and retinal nerve fiber layer thickness measured by Cirrus HDOCT. Arch Ophthalmol 2012;130:312-8.

19. Mwanza JC, Oakley JD, Budenz DL, et al. Macular ganglion cell-inner plexiform layer: automated detection and 
thickness reproducibility with spectral domain-optical coherence tomography in glaucoma. Invest Ophthalmol Vis Sci 2011;52:8323-9.

20. Kim KE, Jeoung JW, Park KH, et al. Diagnostic classification of macular ganglion cell and retinal nerve fiber layer analysis: differentiation of false-positives from glaucoma. Ophthalmology 2015;122:502-10.

21. Jeong JH, Choi YJ, Park KH, et al. Macular ganglion cell imaging study: covariate effects on the spectral domain optical coherence tomography for glaucoma diagnosis. PLoS One 2016;11:e0160448.

22. Jeoung JW, Choi YJ, Park KH, Kim DM. Macular ganglion cell imaging study: glaucoma diagnostic accuracy of spectral-domain optical coherence tomography. Invest Ophthalmol Vis Sci 2013;54:4422-9.

23. Leung $\mathrm{CK}$, Ye C, Weinreb RN, et al. Impact of age-related change of retinal nerve fiber layer and macular thicknesses on evaluation of glaucoma progression. Ophthalmology 2013;120:2485-92.

24. Araie M, Saito H, Tomidokoro A, et al. Relationship between macular inner retinal layer thickness and corresponding retinal sensitivity in normal eyes. Invest Ophthalmol Vis Sci 2014;55:7199-205.

25. Ueda K, Kanamori A, Akashi A, et al. Effects of axial length and age on circumpapillary retinal nerve fiber layer and inner macular parameters measured by 3 types of SDOCT instruments. J Glaucoma 2016;25:383-9.

26. Budenz DL, Anderson DR, Varma R, et al. Determinants of normal retinal nerve fiber layer thickness measured by Stratus OCT. Ophthalmology 2007;114:1046-52.

27. Myers CE, Klein BE, Meuer SM, et al. Retinal thickness measured by spectral-domain optical coherence tomography in eyes without retinal abnormalities: the Beaver Dam Eye Study. Am J Ophthalmol 2015;159:445-56.

28. Song WK, Lee SC, Lee ES, et al. Macular thickness varia- tions with sex, age, and axial length in healthy subjects: a spectral domain-optical coherence tomography study. Invest Ophthalmol Vis Sci 2010;51:3913-8.

29. Zhang C, Tatham AJ, Weinreb RN, et al. Relationship between ganglion cell layer thickness and estimated retinal ganglion cell counts in the glaucomatous macula. Ophthalmology 2014;121:2371-9.

30. Demirkaya N, van Dijk HW, van Schuppen SM, et al. Effect of age on individual retinal layer thickness in normal eyes as measured with spectral-domain optical coherence tomography. Invest Ophthalmol Vis Sci 2013;54:4934-40.

31. Jonas JB, Muller-Bergh JA, Schlotzer-Schrehardt UM, Naumann GO. Histomorphometry of the human optic nerve. Invest Ophthalmol Vis Sci 1990;31:736-44.

32. Varma R, Skaf M, Barron E. Retinal nerve fiber layer thickness in normal human eyes. Ophthalmology 1996;103:21149.

33. Rao HL, Kumar AU, Babu JG, et al. Predictors of normal optic nerve head, retinal nerve fiber layer, and macular parameters measured by spectral domain optical coherence tomography. Invest Ophthalmol Vis Sci 2011;52:1103-10.

34. Bowd C, Zangwill LM, Blumenthal EZ, et al. Imaging of the optic disc and retinal nerve fiber layer: the effects of age, optic disc area, refractive error, and gender. J Opt Soc Am A Opt Image Sci Vis 2002;19:197-207.

35. Kim NR, Kim JH, Lee J, et al. Determinants of perimacular inner retinal layer thickness in normal eyes measured by Fourier-domain optical coherence tomography. Invest Ophthalmol Vis Sci 2011;52:3413-8.

36. Jonas JB, Schmidt AM, Muller-Bergh JA, et al. Human optic nerve fiber count and optic disc size. Invest Ophthalmol Vis Sci 1992;33:2012-8.

37. Quigley HA, Coleman AL, Dorman-Pease ME. Larger optic nerve heads have more nerve fibers in normal monkey eyes. Arch Ophthalmol 1991;109:1441-3. 